\title{
Is the Ontological Approach for the Grade Concept a Necessity for Successful Product Profitability Calculation in a Paper Mill?
}

\author{
Jorma Mattila ${ }^{\mathrm{a}, \mathrm{b}}$, Timo Tiihonen ${ }^{\mathrm{b}}$, Virpi Hotti ${ }^{\mathrm{b}}$ and Martti Juhola ${ }^{*, \mathrm{c}}$ \\ ${ }^{a}$ Honeywell Ltd, Kuopio, Finland \\ ${ }^{b}$ Department of Computer Science, University of Kuopio, Finland \\ ${ }^{c}$ Department of Computer Sciences, University of Tampere, Finland
}

\begin{abstract}
This article creates a generic grade concept that is a synthesis of wide range of terminologies linked with different paper types produced in paper mills. Nearly all paper mills and their system providers define the type and concepts of paper in different ways. The starting point in the grade concept is that it supports the diversified and reliable product profitability calculation as much as possible. Due to the complex nature of grade concept in the paper mills, ontology is used to achieve formal and explicit format for its definition. The formality is understood as an exact description of the concept, which in this article means a mathematical model for the product profitability calculation. The explicit approach means that the concept can be run in a computer by using the third normal mode entity relationship charts (ER). The functionality of the equations is demonstrated by a numerical example.
\end{abstract}

Keywords: Ontology, product profitability computation, paper mill.

\section{INTRODUCTION}

One of the main aims of this article is to create consistent and compact terminological grade concept for the paper mills. This would define the grade concept explicatively as well as the terms and synonyms used for the grade concept. It is problematic to standardize the grade concept as one paper mill uses terms that may have different meaning in another paper mill. The grade concept, as well as other concepts, can be understood through complications. If one understands the problem, one can also figure out that the grade, as a philosophical concept, cannot be given concepts that are comprehensive, definite and easy to understand. This is due to the general openness of the concepts as well as missing traditions in the grade operation, or that the traditions are reasonably young. The vague grade concept has complicated the production efficiency and calculation of the economic effectiveness linked with the grades, as well as the comparison of cost-effectiveness between different paper machines and paper mills.

The other aim for the grade concept is that the terms of definition can be checked afterwards, which allows adding new concepts or removing unnecessary ones. The principal starting point is the need to clarify the grade concept and standardise the terms used in a way that they take into account the needs of IT. The meaning of the concept is vital in creating the IT solutions, among other things, as follow:

- $\quad$ The daily work is based on usage of IT,

*Address correspondence to this author at the Department of Computer Sciences, 33014 University of Tampere, Finland; Tel: +358-3-35517972; Fax:+358-3-35516070; E-mail: Martti.Juhola@cs.uta.fi
- $\quad$ IT systems have become more and more important tool for leadership and communication that requires consistent terminology in the paper mills, their customers and the system providers,

- $\quad$ The integration of the applications in the IT system is not possible without common grade concept.

The optimization of a paper mill's product range and the product specific profitability have been tried to calculate for decades; however, usually they have either failed or not fulfilled the expectations. The reasons for the failures have been the vagueness of the grade concept, inaccuracy of data linked with the consumption measurements in raw materials and the algorithmic problems with the product range optimization [1]. Even though a paper mill represents traditional industry, the grade concept with all its variables is a quite new terminology and, therefore, somehow unclear and without generally accepted standard.

In this article a product profitability calculation means calculation that is based on the actual measurements collected from the paper production process, and which allows the development of grades, products and product range to be controlled even in short period of time. The product profitability calculation differs from the accounting profit calculation in a way that it is not based on the procurement and sales documents. It is based on the actual process and production activities. The profit calculation based on the accounting records cannot react quickly enough to the product related profitability. It can be used for identifying how well the mill is doing on a monthly, quarterly or yearly basis [1].

The production grade means paper that has certain specifications. The specification is defined by a paper machine, a finishing machine and their machine conditions, as well as 
the raw materials used and their relations. A run means a batch manufactured by following a certain specification. The grade variant means different concepts of the grade concept in this article. There is always some kind of connection between the grade variants. If the relationship between the variants is, described by using an IT term, a many-to-many, $(n: n)$ relationship, it causes problems when cumulating consumption, costs and product profitability from a variant to another. When comparing the product profitability, the criteria are margin per ton and margin speed (margin per hour) [1].

Together with the grade, the most central terms in the product profitability calculation are an order and a run. The order clarifies what needs to be manufactured, when and by which price paper is sold. One run may produce several orders of the same grade. Therefore, the orders in the same run will have the same production costs. The price of the orders is conducted by the profit margin that can be different, regardless of the same production costs. If the same order is manufactured in several different runs, then the production costs will be different on the same order depending on the runs, which a part of the order has been produced. The cost of one order is an accumulation of several runs. However, a problem may occur in how to calculate the profit margin in a reliable way for the whole order and, in addition, the profit margin for all of the customer's orders. A similar problem occurs when the grade variants' profit margins are calculated - especially, if the grade variants do not have a one-to-many relation $(1: n)$.

Traditionally, a weighted average is used when calculating and cumulating the product profitability. Here a hypothesis is created that when modeling the product profitability calculation by using the methods in the IT and ontology, one can calculate the profit margin in a cumulative way without the weighted average for the run, order, grade and different grade variants. It is often difficult and sometimes even impossible to verify the right weighted average. The problem is emphasized when the margin per ton is changed to the margin per hour by using the production efficiency (ton/hour). Together with the margin speed, the production efficiency is the most important technical indicator when assessing the paper machine's productivity. It is calculated by dividing the net tons by time that has lapsed for manufacturing the tons in question on the paper machine.

The run and grade concepts introduced here are suitable for mills that manufacture paper for newspapers. This is because the grade remains the same from the paper machine to the roll wrapping. If the mill has separate coating machines, the grade of the base paper will change into coated paper. In this instance, the grade concept needs to expand from the grade of base paper to the grade of the final product. This article is based on the assumption that when the product profitability is calculated, the grade remains unchanged throughout the production chain.

The actual application i.e. the grade concept in a paper mill and the real time product profitability calculation only have few comprehensive publications. The approach taken here in which the data model is improved by ontology is used in the reference [2].
Next chapter will define ontology and ontological terminology. Chapter 3 will describe the concept model linked with paper grade from the ontological point of view. Chapter 4 will solve the cumulative values of product profitability based on the theory in chapter 3 . Chapter 5 will create a mathematical model for cumulative product profitability. Chapter 6 will utilize the hypothesis of the mathematical model by going through some ranking lists showing product profitability.

\section{ONTOLOGY AND GRADE CONCEPTS IN PAPER MILL}

A hierarchy of concepts is one of the most important components of thesauri and classification systems where the concepts have more general or specific meanings. At recent times, many thesauri and classification systems have been developed towards ontology in order to provide more automated information processing [3].

The term "ontology" has been used for many different purposes, which have caused confusion and lack of clarity [4]. One of the widely used general definitions is that "ontology is a formal, explicit specification of a shared conceptualization" [5]. Formal means that ontology should be machine-readable. Explicit refers to the idea that concepts and constraints of ontology are explicitly defined. Shared means that ontology captures consensual knowledge accepted by a group instead of an individual. Conceptualization refers to an abstract model that belongs to a scope from which ontology has developed. This model identifies the relevant concepts of its scope.

Ontological analysis is the first step towards an effective knowledge representation system. It clarifies the structure of knowledge. With ontological analysis, it is possible to develop a vocabulary that represents the knowledge of a specific domain. In ontology, terms, concepts and relations can be combined in a way that an essential conceptual structure of the domain is captured. This knowledge can be shared with others who have similar needs for knowledge representation in the same domain. Advantaging shared ontology eliminates the need to replicate the knowledge-analysis process [6].

Behind ontological analysis is a terminology work that is an interdisciplinary subject field for ordering and transferring knowledge. The terminology work is based on analysis and structuring of concepts and relations. Fig. (1) clarifies relations between object, concept, term and definition that are essential elements of the terminology work [7].

Objects can be abstract or concrete, characterized by properties. These properties are abstractions called characteristics and they form a unit called a concept. The concept can be individual or general. An individual concept is connected to a single object, and a general concept is connected to several similar objects. Because concepts are abstractions, terms and definitions are needed for communication. Terms are expressions used to refer to concepts, and definitions are statements describing the concept [7].

Concepts are always related to other concepts and form concept systems. A concept analysis is a terminological 


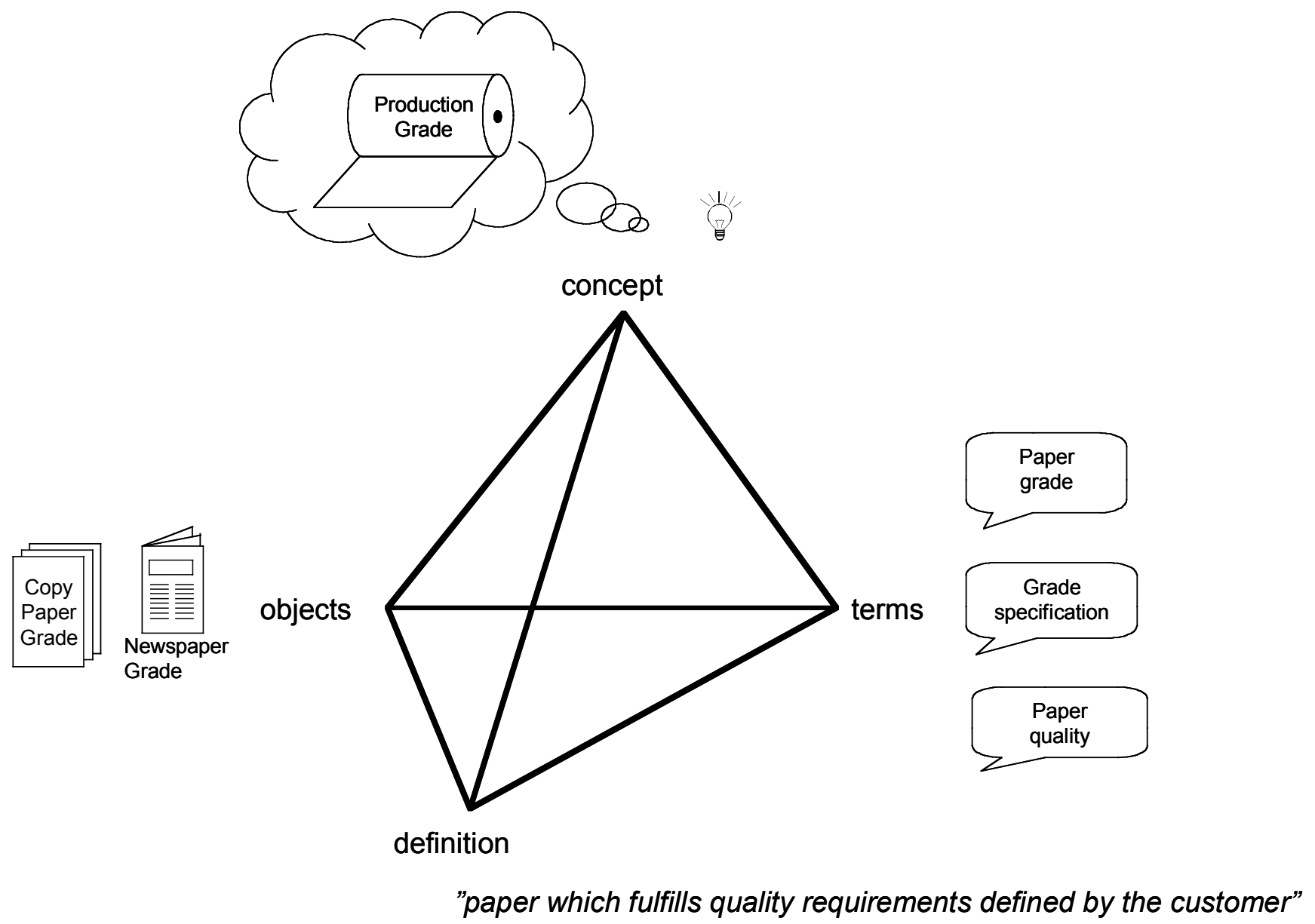

Fig. (1). Object, concept, term and definition (modification of Suonuuti, 2001).

work process where relations among concepts are clarified and concept systems formed. In practical terminology, three types of relations are identified: generic, partitive and associative (Fig. 2). A generic relation exists between the subordinate and super ordinate concept. For example, a newspaper grade is a subordinate concept to a production grade. In partitive relation, subordinate concepts are parts of the wholeness formed by the super ordinate concept. For example, a newspaper grade cost is composed of many different manufacturing costs. Associative relations contain different concept relations that cannot be classified as generic or partitive relations. For example, relations between paper grades and products at the market are associative [7].

Technologies and applications based on ontology have been developed recently [8], among others the finalization of ER charts' terminology. A good example of an application using ontology is so called Semantic Web in which the semantics is modeled for the usage of machines instead of traditional vocabulary that are created from human point of view [9]. The Semantic Web uses terminology understood by machines and the relations between them [10].

\section{CONCEPTUAL DEFINITION OF PAPER GRADES}

Grade is an example of the special nature of the concept world in a paper mill. Grade is not a product nor is a product a grade; however, there is no product without grade. There is clearly an associative relation between product and grade. Based on the experience in several production management projects the authors of this study have faced the same problem over and over again: how to define the grade concept in a way that it is possible to use the definition both in multileveled operative as well as decision making functions.

There are several grade variants and their possible combinations. As an example of grade variant combinations one can mention products that are exported to a certain country. As addition, one should be able to calculate the profitability of any variant and the variants' combinations. The calculation will be more successful if:

1. the grade concept is clear,

2. many-to-many relation is broken into one-to-many relation, and

3. consumption, cost and profitability data is saved close enough to the action level from which they can be cumulated to grade variants and their free combinations.

Fig. (3) shows a concept model of an application ad hoc entity model based on product profitability. The rectangles present the entities and the ovals represent the consumption and cost data of the target. The relations are marked as follow: 


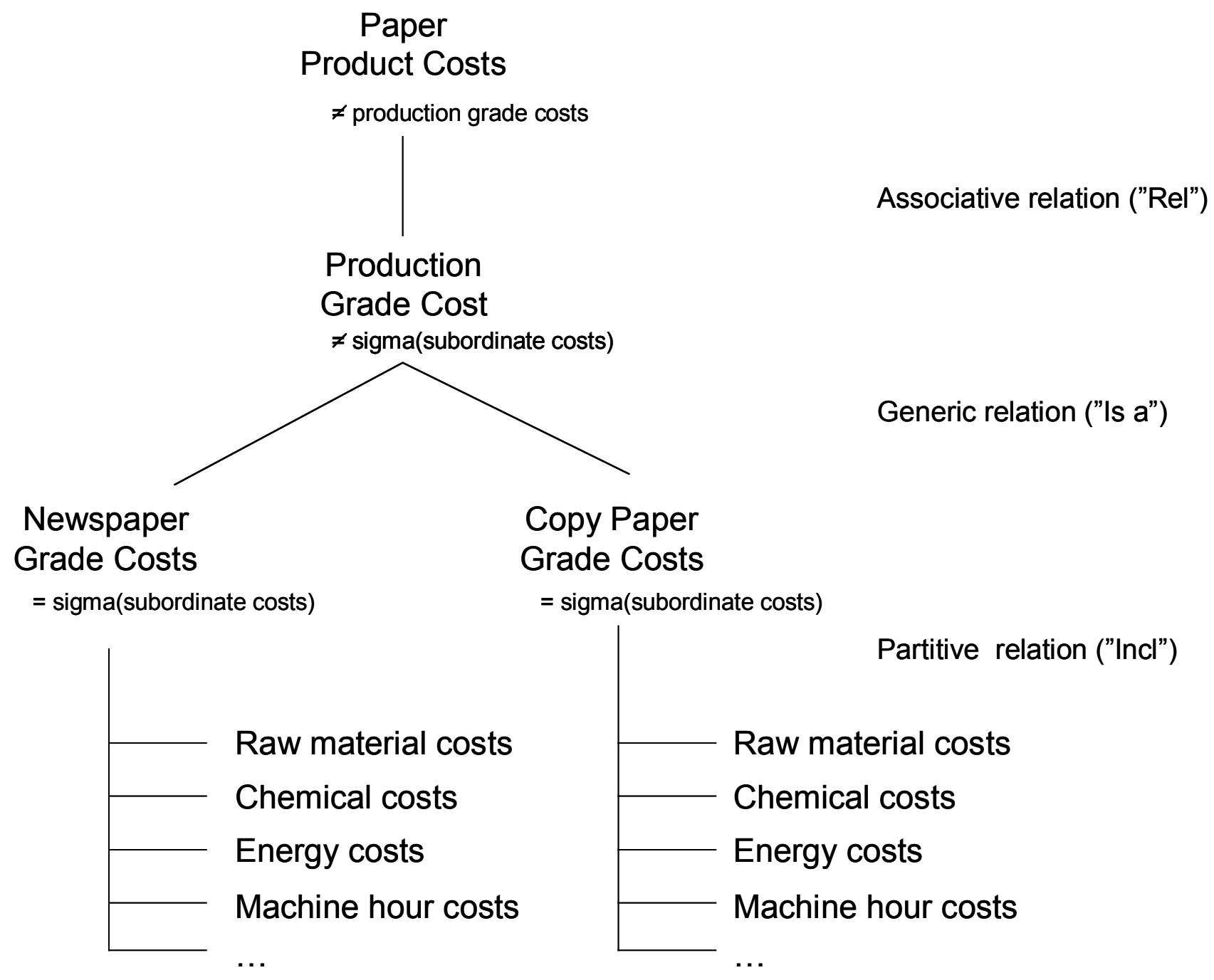

Fig. (2). Three types of relations.

$(1,1)-(0, n) \quad$ The relation is a one-to-many relation, where many varies from zero to $n$. If zero is one (1), many varies from one (1) to $n$,

$(0, n)-(0, n) \quad$ The relation is a many-to-many relation, where many varies from zero to $n$. If zero is one (1), many vary from one (1) to $n$.

It is assumed that the machine and customer rolls' consumption and cost data as well as the run's efficiency data are known. The task is to calculate margin per ton and margin speed for customer, order, product and grade. The problem is many-to-many relation between product and grade. If the order is run in several runs, that is perfectly viable, then the model does not represent reality any more. The ad hoc model is clearly inadequate for product profitability calculation. To improve the model ontology may be used to solve the problem. Then the relations between the concepts and meanings are better clarified than by traditional entity model [2].

Fig. (4) shows the grade concept from the ontological point of view. The grade concept can be divided into terms used by sales and production. In sales, the relevant terms are product, sales grade, end-use and printing method. The terms in production are machine, machine line, production grade, recipe and run. The relations linking notations have been described by using different line types that are explained in the Legend section of Fig. (4). Table 1 lists the most relevant terms.

Each run represents one production grade on a paper machine. Grade means paper that is on the machine roll and it fulfills the quality requirements set by the customer. Quality of the paper on the paper machine is achieved by the machine settings and the raw material combinations based on the recipe. As examples of the machine settings, can be given the usage of Yankee cylinders and nip pressures. Production grade is, therefore, a special grade definition that includes the quality requirements of the customer as well as the control parameters of the paper machine. The exact quality and technical production instructions are defined to product range in a run. These instructions are called run standards and they are a part of the standard recipe. Run standards are defined for production grade and they are specified on each run based on the quality requirements given by the customer. The same run may have several customers and orders as long as the paper ordered by the customer is the same production 


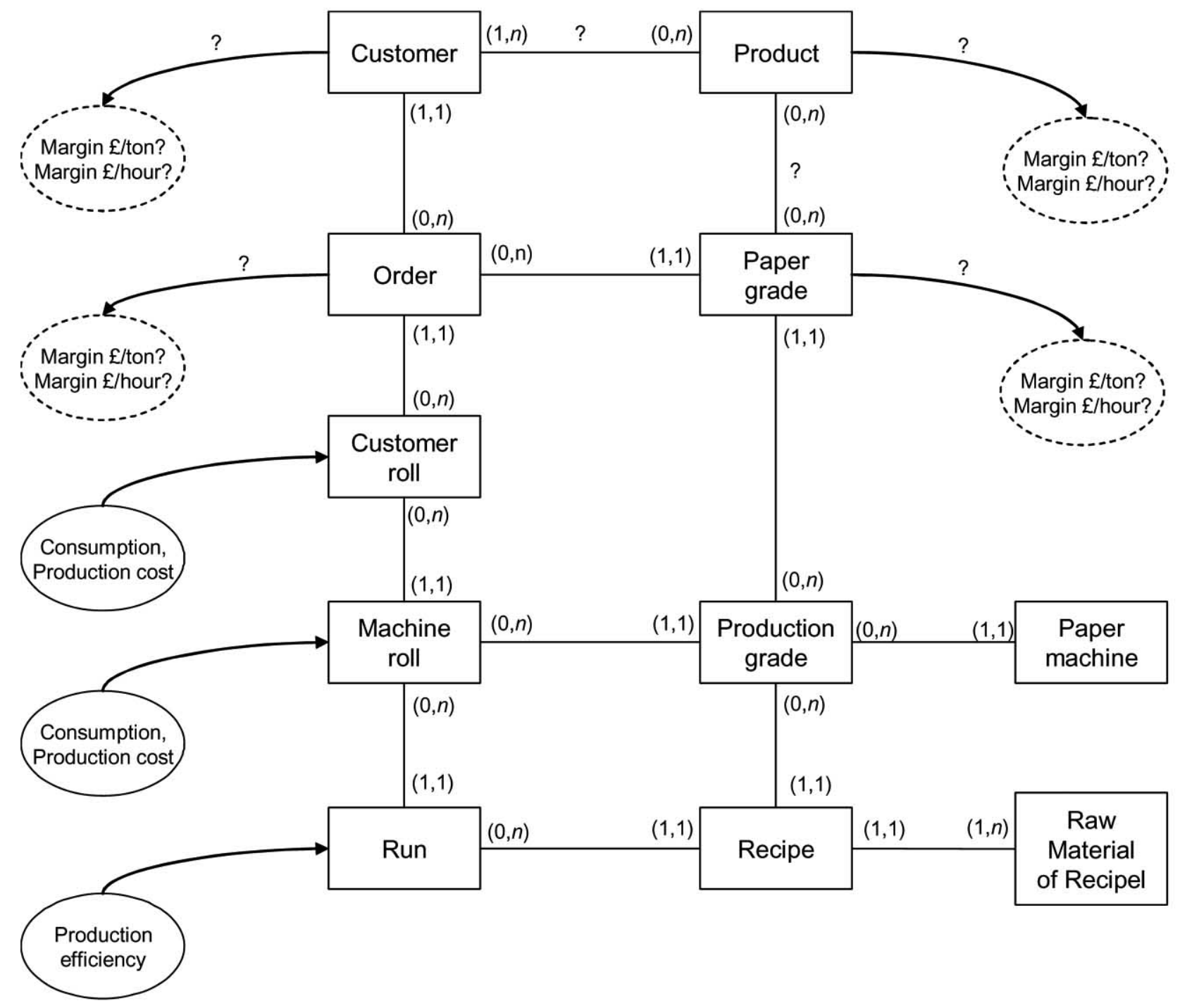

Fig. (3). Grade and its variants' ad hoc entity model.

grade - the sales grade, product, end usage or the printing method do not need to be the same.

The markets operate by product items. A product is a generic name that all paper mills use for similar paper types. Examples of product items are as follow: newsprint, coated art paper and polished magazine paper. After sales have sold a product to a customer, the paper mill concludes what are its own sales and production grades based on the customer, bases weight, print method and product. The customer and sales grade also define the end usage of paper as well as the printing method. The end usage means the application of paper and the printing method defines the type of printing the paper is meant for. The examples of the end usage are catalogue and bible papers and of printing methods offset and gravure printing.

Next, ad hoc entity model is compared with the ontology description. By looking at the relations between grade variants and the correlation between run, order and product grade one can realize that the accuracy of ad hoc model is not enough for formal and explicit entity model (Fig. 5). To modify the model to the third normal mode, the order needs to be divided into order rows because each row can represent a different production grade and, therefore, they can be manufactured on different runs as well. This is the reason why the model needs an order row for each run. Another significant adjustment is between the production grade and the product. The many-to-many relation needs to be broken down into one-to-many relation. This is best achieved by storing the reference keys of each grade variants needed in order. The reference keys are, for example, product, sales grade and paper grade. After a run, a customer roll is created for an order row. This roll can be linked to the relevant grade variants by using one-to-many relation. In a similar way, a customer roll can be linked with the important reference keys for the profitability calculations that are among others recipe, paper machine and machine line.

Based on the entity model and the ontology one can define the existence of the following relations: (1) Run repre- 


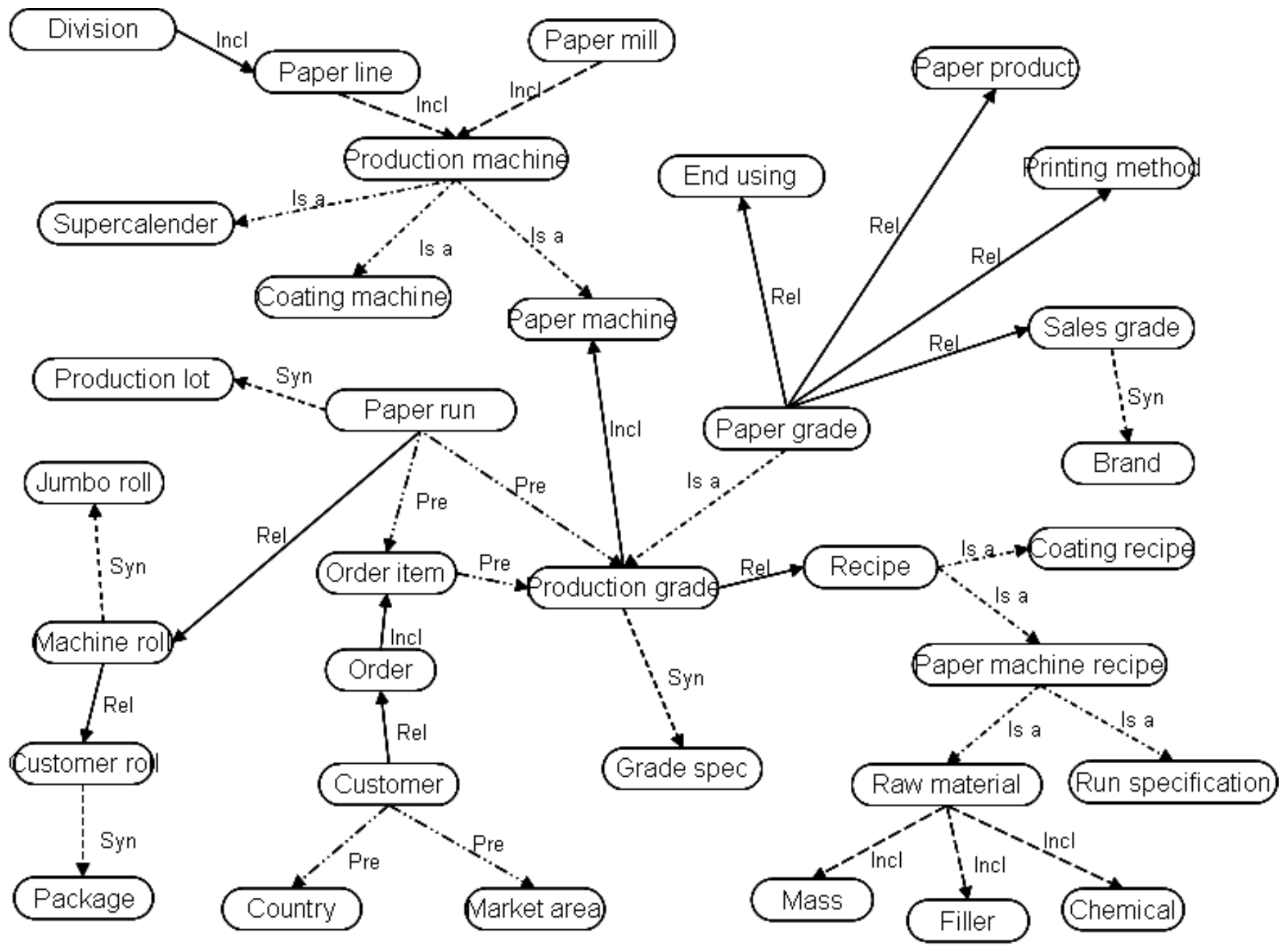

Legend

Relationships:

Constrains:

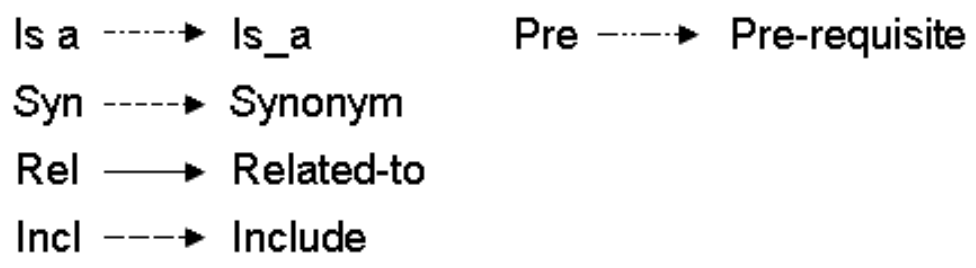

Fig. (4). Grade variant domain ontology.

sents always one production grade. The same production grade can be manufactured on several runs. The model shows that the same order row can be manufactured on several runs and a run may have several different order rows. (2) An order consists of several order rows. An order row is filled with production grade that defines which of the paper machines is used for manufacturing the order. The same production grade exists on several order rows. (3) Recipe defines how the production grade is manufactured on a certain paper machine. Recipe for raw materials includes some raw materials. The same raw material recipe can be used for manufacturing several production grades. (4) Production grade depends on the paper machine, as does the recipe. A paper machine can be used for running several production items. (5) By running the same grade on several paper machines, the production grade defines how the grade is run on that specific paper machine. (6) One grade can be run on several paper machines and, correspondingly, one paper ma- 


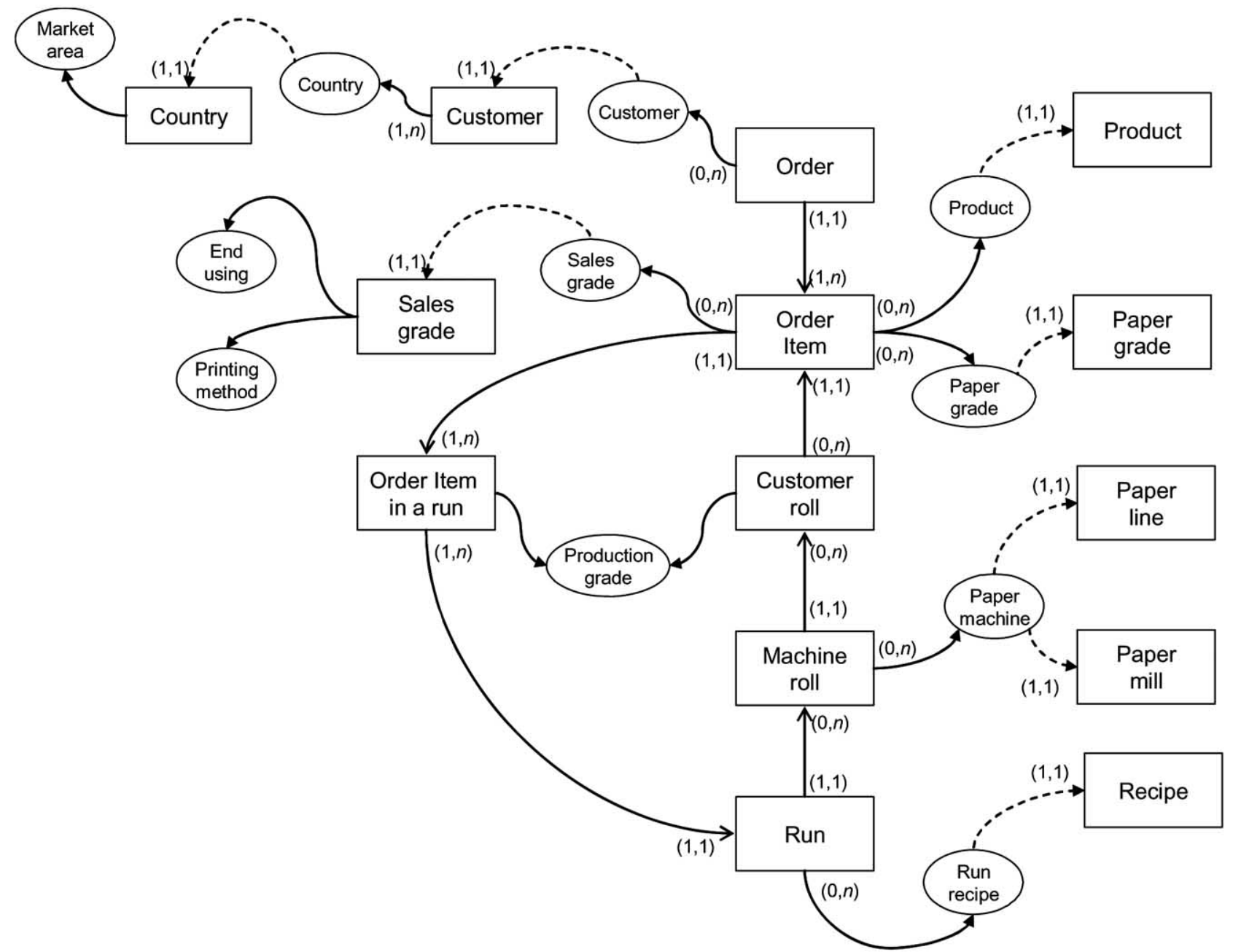

Fig. (5). Grade concept's entity model based on product profitability calculation, specified by ontology.

chine can used to run different types of grade. (7) A customer orders several grades and, similarly, the same grade is produced for more than one customer. (8) The same grade can be sold as several sales grades and a sales grade may contain more than one grades. (9) Sales grade defines the printing method. The same printing method may include several sales grades. There is a many to many relation between sales grade and production grade. The relationship is clearly associative. (10) Sales grade defines the end usage and the same end usage may come from different sales grades. (11) There is no unambiguous relation between product and sales grade. (12) A run produces machine rolls. Typically, a machine roll belongs to a run. (13) Customer rolls are cut from the machine rolls by using a pattern. A customer roll usually belongs to one machine roll. (14) The customer roll is connected to the order during the manufacturing. One order row consists of several customer rolls. (15) The price information is stored in the order row during the sale phase. (16) A paper machine is always a part of a real paper mill and that mill may have more than one paper machines. (17) A paper machine belongs to a machine line. The machine line may have several paper machines. (18) A machine line belongs to a division and a division includes several machine lines. (19) A customer belongs to a country and that country has several customers. (20) Raw material consumption data can be calculated per a machine roll. (21) Production cost can be calculated for a run. (22) Production efficiency data can be calculated for a run. (23) One run may have several order rows and the same order row can be produced in several runs. (24) An order induces order rows. (25) The raw material recipe includes raw materials. The same raw material can be used in several recipes.

One can see from the relations that there is a many to many relationship between the product and the product grade, as well as between the product and the sales grade; and the variant and the customer. Therefore, one cannot cumulate the expense data of a run directly to the sales grade, product or customer.

\section{CUMULATIVE NATURE OF THE GRADE VARI- ANTS' PROFITABILITY DATA}

As one can see from the grade's conceptual definition, a part of the grade variants is many to many relations. Even if one could calculate profitability for a run and the production grade, one problem would remain how to calculate 
Table 1. Grade Variant Domain Ontology

\begin{tabular}{|c|c|c|c|}
\hline Concept & Synonym & Description & Related to \\
\hline \hline Production grade & Grade spec & To be run grade & Recipe, paper machine \\
\hline Paper run & Production lot & Manufacturing lot & Machine roll, customer roll \\
\hline Order item & & Paper roll width & Order, customer roll \\
\hline Recipe & & Raw material mix & Raw material, production grade \\
\hline Machine roll & Jumbo roll & Roll from paper machine & Run, customer roll \\
\hline Paper grade & & Paper usually & Product, sales grade, end using \\
\hline
\end{tabular}

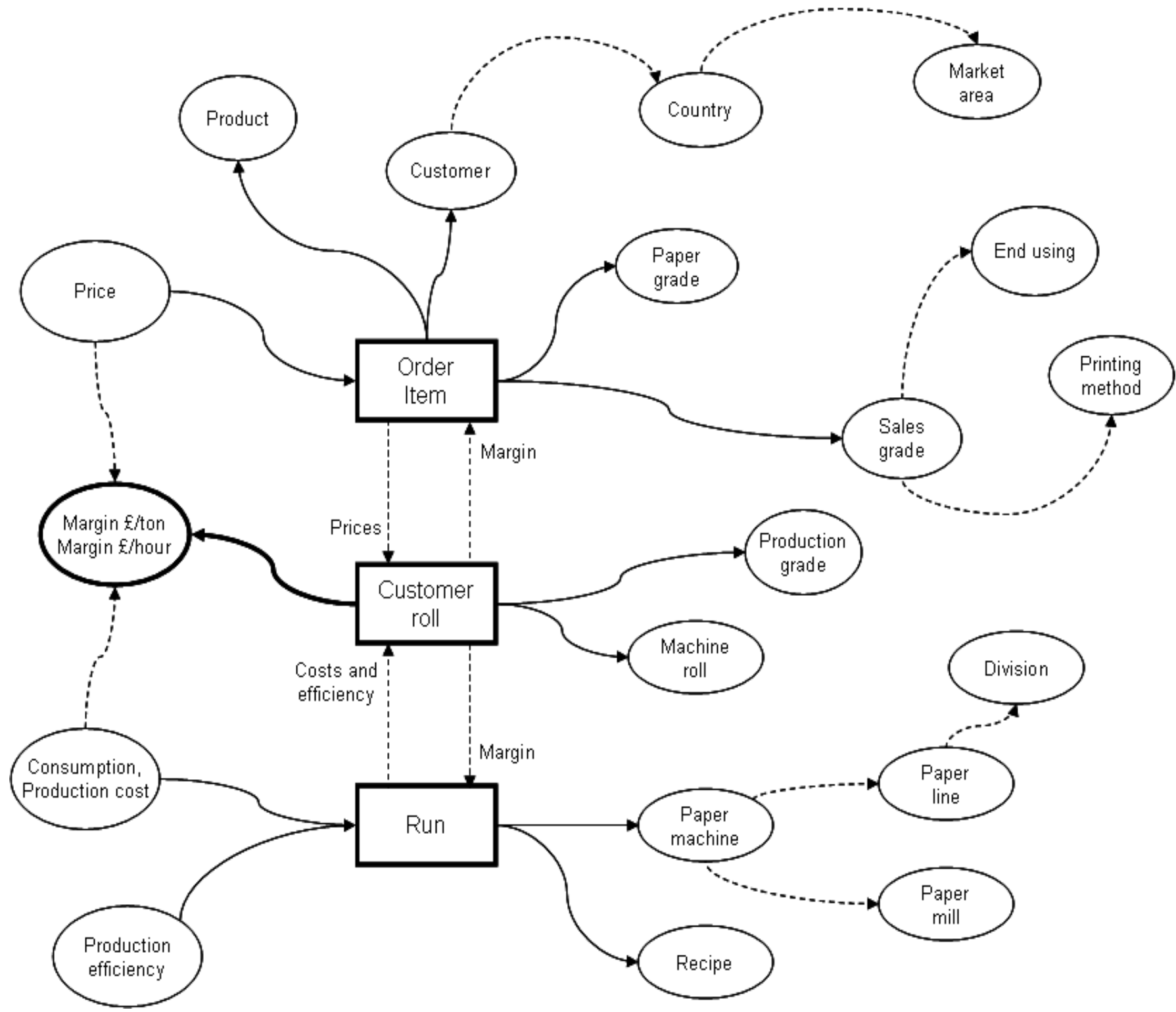

Fig. (6). Graphical description of the solution of many-to-many relation problem in which an order row represents the associative relation of the grade variants.

profitability for those variants, which do not have an unambiguous relation with the product grade. As examples, one can mention sales grade and product. Another problem is how to calculate the profitability of an order, if the order has been manufactured in more than one run; or how to calculate the profitability and suitability of a customer to the precise paper mill. The next Fig. (6) shows a solution to these questions. There the order row acts as an integrator, i.e. the order 
row stores those grade variants' codes that are not one-tomany relation against the run and the production grade. The representatives of these grade variants are the product and the sales grade. The customer and the sales price of the product have been stored in the order row during the sales phase.

In the notation of Fig. (6), the solid line represents the derived data and the dotted line the data linked with the derived data. The derived data means, for example, that the customer data includes the data of country and the market area. After the product, sales grade and customer data are stored in the order row, one can add the same data to the customer roll after a run. The customer roll includes the production grade data. The derived data (country, printing method, end usage and recipe) can also be stored in the customer roll. Through the machine roll and the run the customer roll will also receive the consumption and cost data and price data comes from the order.

As a rule, the customer rolls are always packed for a specific order in a paper mill to be able to store the following data on the customer roll: physical directions, weight, order row, customer, country, market area, and product, sales grade, printing method, end usage, run, grade, production grade and recipe. All except physical directions and weight are needed for the calculation of profitability.

As the customer roll has the data listed in the previous paragraph, the consumption, cost, margin per ton and margin speed can be cumulated directly to any of this data and free combinations of data by using, for example, OLAP multidimensional cubes (OLAP stands for On-line Analytical Processing) [11].

\section{CUMULATIVE EQUATIONS FOR PRODUCT PROF- ITABILITY}

This chapter describes the cumulative nature of the margin calculation as mathematical equations. The starting assumption is that the consumption and margin per each customer roll has been calculated already [1]. Therefore, one can assume that the margin of a customer roll, $R_{r}$, and weight $N_{r}$, as well as the net efficiency of the run $E^{a}$, and the operating time $T^{a}$ are known. If the customer roll's $r$ data contains the information of run $(a)$, order $(o)$, production grade $(g)$ and recipe $(c)$ and machine line $(l)$, one can calculate their margin per ton and margin speed by using the equations (1) $-(10)$.

The total margin of a run $M^{a}$ [\$/run] and the net production $P^{a}$ [ton/run] is received by adding up all of those customer rolls that are a part of a run $a$, i.e. the margin is calculated as follow

$M^{a}=\Sigma_{r} R_{r}^{a}$

and the net production

$P^{a}=\Sigma_{r} N^{a}{ }_{r}$.

The equations (1) and (2) can be used to calculate the margin per ton $Q^{a}$ [\$/ton] for a run per each net ton $P^{a}$ :

$Q^{a}=M^{a} / P^{a}$.
The margin speed of a run $S^{a}$ [\$/hour] is defined by multiplying the margin per ton $Q^{a}$ by the run's net efficiency $E^{a}$ [ton/hour]

$S^{a}=Q^{a} \cdot E^{a}$.

If the order's net production $o$ in a run $a$ is $N^{a o}$, one can calculate the order's $o$ margin per ton $U^{a o}$ in a run $a$ by adding up the margin $R^{a o}$ of the runs in the order and dividing the sum by the net weight $N^{a o}$

$U^{a o}=\left(\Sigma_{r} R^{a o}{ }_{r}\right) / N^{a o}$.

The run $a$ has the order $o$. When calculating the margin speed $Z^{a o}$ one needs to take into account that all the orders in that run needs to use the run's net efficiency $E^{a}$

$Z^{a o}=U^{a o} \cdot E^{a}$.

If the order is manufactured in several runs, the order's margin per ton can be cumulated. Then the margin $R^{o}$ of the rolls in the order is summed over runs and the sum is divided by the corresponding net weight of the rolls $N^{\circ}$. By using this method, one avoids using the weighted average.

$U^{o}=\Sigma_{r} R_{r}^{o} / \Sigma_{r} N^{o}$.

The margin speed of an order is calculated by cumulating the rolls' margin per ton over the runs $R_{a}{ }^{\circ}$ and the net weights of the respective runs $N_{a}^{o}$ to calculate the margin per ton. The net weights $N_{a}$ and the operating times $T_{a}$ are used to calculate the runs' efficiency. The sums received this way are divided to reach the following equation:

$Z^{o}=\left(\Sigma_{a r} R_{a r}^{o} / \Sigma_{a r} N_{a r}^{o}\right) \cdot\left(\Sigma_{a} N_{a} / \Sigma_{\mathrm{a}} T_{a}\right)$

The production grade's $g$ margin per ton $G^{g}$ is reached by adding up the grade's margins $M^{g}$ and respective runs' net weights $N^{g}$ from those runs that has been used to manufacture that grade. Then, the sums are divided. When calculating the grade's margin per ton one needs to take into account the time period used

$G^{g \Delta t}=\Sigma_{a} M^{g \Delta t}{ }_{a} / \Sigma_{a} P^{g \Delta t}{ }_{a}$,

where $\Delta t$ defines the time period in question.

Analogically, the margin speed $F^{g}$ of a production grade $\mathrm{g}$ is calculated by using equations (8) and (9):

$F^{g \Delta t}=\left(\Sigma_{a} M^{g \Delta t}{ }_{a} / \Sigma_{a} P_{a}^{g \Delta t}\right) \cdot\left(\Sigma_{a} N^{g \Delta t}{ }_{a} / \Sigma_{a} T_{a}^{g \Delta t}\right)$.

Based on equations (9) and (10) the runs are manufactured by a period of time $\Delta t$ and production grade $g$ is manufactured.

The margin for recipe and machine line is calculated in a similar way as production grade, as a run uses one recipe and one paper machine only.

The margin of sales grade, end usage, printing method, product, country and market area, as well as any of these variables' combination, is calculated in a similar way as in equations of the order (5), (6), (7) and (8), when the order index $o$ is replaced with any of the grade variants listed in this paragraph.

Example: Let us assume that the runs are $a 1, a 2$ and $a 3$. Runs $a 1$ and $a 2$ produce a concept $g l$ and run $a 3$ a concept g3; these are the same sales grade based on the order. As addition, the run has two orders $o 1$ and $o 2$. The following numerical assumptions are valid: 


$\begin{array}{llll}\text { customer roll } & N_{l}^{a l, g l, o l}=1.000 \text { ton } & \text { and } & \operatorname{margin}{R_{l}}^{a l, g l, o l}=100.00 \$ / \text { roll, } \\ \text { customer roll } & N_{2}^{a l, g l, o 2}=0.500 \text { ton } & \text { and } & \operatorname{margin}{R_{2}}^{a l, g l, o 2}=80.00 \$ / \text { roll, } \\ \text { net efficiency } & E^{a l, g l}=20 \text { ton/hour } & \text { and } & \text { duration } T^{a l, g l}=0.075 \text { hour, } \\ \text { customer roll } & N_{l}^{a 2, g l, o l}=1.000 \text { ton } & \text { and } & \operatorname{margin}{R_{l}}^{a l, g l, o l}=120.00 \$ / \text { roll, } \\ \text { customer roll } & N_{2}^{a l, g l, o 2}=0.500 \text { ton } & \text { and } & \operatorname{margin}{R_{2}}^{a l, g l, o 2} \text { on } 90.00 \$ / \text { roll, } \\ \text { net efficiency } & E^{a 2, g l}=15 \text { ton/hour } & \text { and } & \text { duration } T^{a 2, g l}=0.1 \text { hour, } \\ \text { customer roll } & N_{l}^{a 3, g 3, o l}=1.000 \text { ton } & \text { and } & \operatorname{margin}{R_{1}}^{a 3, g 3, o l}=90.00 \$ / \text { roll, } \\ \text { customer roll } & N_{2}^{a 3, g 3, o 2}=0.500 \text { ton } & \text { and } & \operatorname{margin}{R_{2}}^{a 3, g 3, o 2}=50.00 \$ / \text { roll and } \\ \text { net efficiency } & E^{a 3, g 3}=18 \text { ton/hour } & \text { and } & \text { duration } T^{a 3, g 3}=0.083 \text { hour. }\end{array}$

By using the equations (1) - (10) the following results are achieved:

By eq. (1) the total margin for runs $M^{a l}=180.00, M^{a 2}=210.00$ and $M^{a 3}=140.00 \$$,

by eq. (2) the net production of runs $P^{a l}=1.5, P^{a 2}=1.5$ and $P^{a 3}=1.5$ tons,

by eq. (3) the margin per ton per run $Q^{a l}=120.00, Q^{a 2}=140.00$ and $Q^{a 3}=93.30 \$ /$ ton,

by eq. (4) the margin speed per run $S^{a 1}=2400.00, S^{a 2}=2100.00$ and $S^{a 3}=1679.40 \$$ /hour,

by eq. (5) the order, ol, margin per ton $U^{a l, o l}=100.00, U^{a 2, o l}=120.00$ and $U^{a 3, o l}=90.00 \$ /$ ton; the order, $o 2$ margin per ton $U^{a l, o 2}=160.00, U^{a 2, o 2}=180.00$ and $U^{a 3, o 2}=100.00 \$ /$ ton,

by eq. (6) the order, ol margin speed $Z^{a l, o l}=2000.00, Z^{a 2, o l}=1800.00$ and $Z^{a 3, o l}=1620.00 \$$ /hour; the order, $o 2$ margin speed $Z^{a l, o 2}=32000.00, Z^{a 2, o 2}=2700.00$ and $Z^{a 3, o 2}=1800.00 \$ /$ hour,

by eq. (7) order, $o 1$ margin per ton over the runs $U^{o 1}=103.33 \$ /$ ton and the order, $o 2$ margin per ton $U^{o 2}=146.67$ $\$ /$ ton,

by eq. (8) order, o1 margin speed over run $Z^{o l}=1802.08 \$ /$ hour and the order, $o 2$ margin speed $Z^{02}=2557.92$ $\$$ /hour when the sum efficiency of runs is 17.44 tons/hour,

by eq. (9) grade's $g 1$ margin per ton $G^{g l}=130$ and grade's $g 2$ margin per ton $G^{g 2}=93.33 \$$ ton, when $\Delta t$ includes both runs,

by eq. (10) grade's $g 1$ margin speed $F^{g l}=22282.20$ and grade's $g 2$ margin speed $F^{g 2}=1679.94 \$$ hour.

\section{RANKING LISTS}

The grade variants' mutual order is described by ranking lists. Figs. (7-9) show as an example three ranking lists. The first one shows a run's margin speed as a function of run time; the second one the grade's margin per ton versus production ton, and the third one the order's margin per ton is compared with the average margin of that particular grade over a certain time period.
Fig. (7) shows margin speed as a function of run time. In this case, it is enough to concentrate gross profit (GP) i.e. only the variable cost is included. One can clearly observe from Fig. (7) the profitable grade variants and those that need further action to become more profitable. One can see that the three last grade variants are uneconomical. Based on the figure, the mill can run further analysis as why some theoretically profitable variants are uneconomical when in production. The reason could be, among others, too short run periods especially when the cost in changing the grade is 


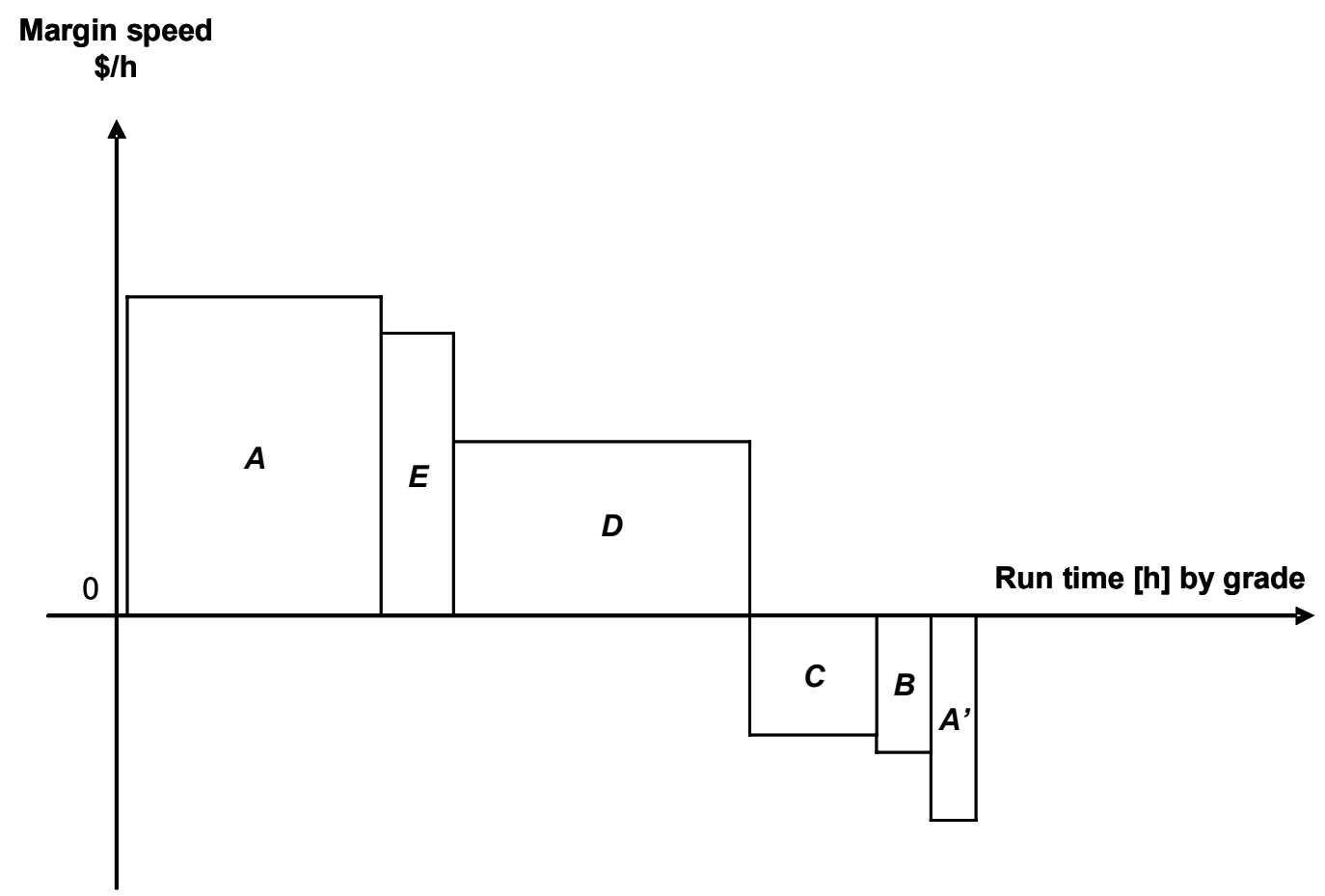

Fig. (7). A ranking list based on margin speed as per run time.

significant. For example, in Fig. (7) the grade variant $A$ becomes profitable by lengthening the continuous run time of the grade (compare $A$ and $A^{\prime}$ ).

In Fig. (8), the ranking list is based on margin per ton. The bars represent the production tons and the dashed line the margin percent of each variant. One can easily define from Fig. (8), what type of paper and how much of it is sold with bad margin. If the production ton and margin are small, the problem is not significant from the overall profitability point of view.

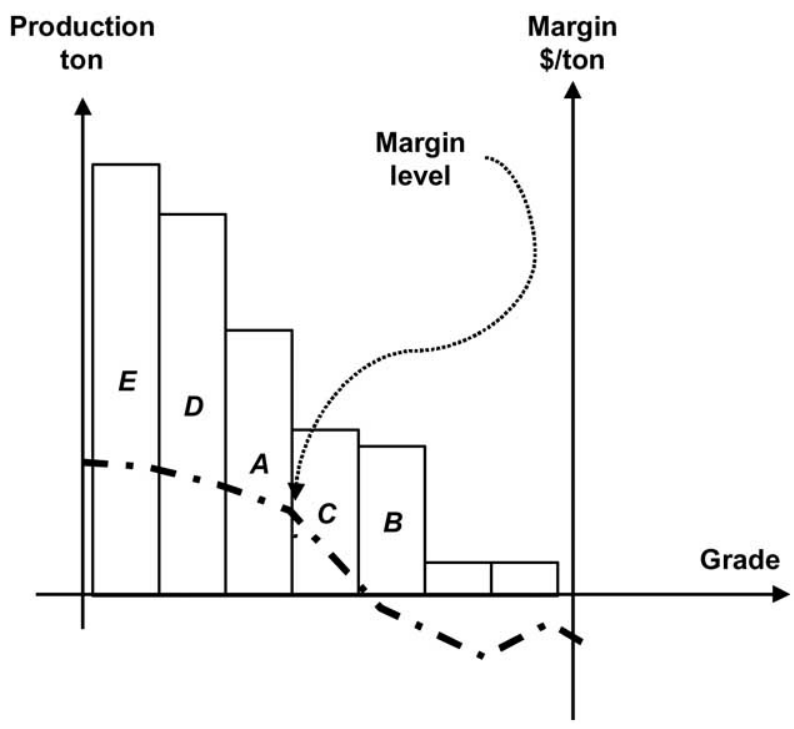

Fig. (8). Grade based ranking list of margin per ton in relation with the grade's production ton.

The ranking list in Fig. (9) clarifies the orders' margin per ton versus the other orders of the same grade. One can see that the order $T 6$ is clearly under the average and the order a significant amount. It seems that the order $T 6$ has a weakening impact to the mill's results. Presumably, it would be worth analyzing it further to see whether one can increase its profitability somehow. If the graph is based on real runs, one could also check the impact of trimming, efficiency and raw material data to the runs of T6. Trimming means here how well the width of the order can be justified against the width of the machine roll to guarantee that the order requirements are fulfilled. This is also called a trimming problem. If the graph is based on data when the order has been accepted, the selling price and the costs of logistics are the only factors that can be used to contribute to the profitability.

\section{CONCLUSIONS}

This article contemplated why the product and grade based profitability calculation in a paper mills causes constant problems. Based on wide project experience, one reason may be the vagueness of the grade concept. Therefore, a concept analysis and ontological study were organized for grades. These studies confirmed that grade concept is multidimensional and difficult to formulate. An additional problem is that concept structure in different paper mills differs a lot from mill to mill. This has made it more difficult to calculate and estimate the profitability of grade variants and, therefore, a unified way to calculate the product profitability has not been developed. Traditionally, the product profitability is calculated based on the standard recipes, in which the margin is mainly production grade [12]. Another problem has the difficulty in cumulating the margin data in the concept space. The paper mills have tried to find out a right way to calculate the product profitability without realizing that the main reason may be in the vagueness of the grade concept and the difficulty in cumulative approach when moving from a grade variant to another. This article has described the 


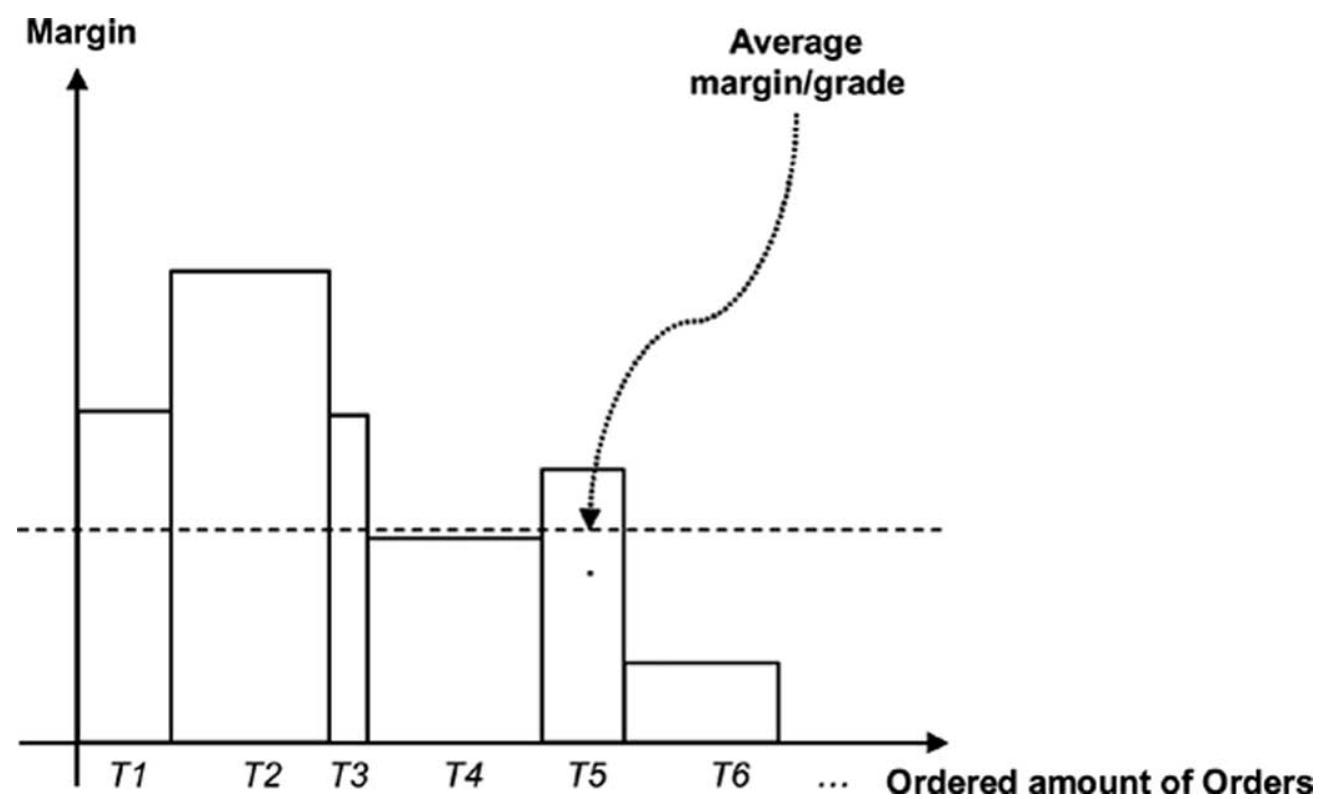

Fig. (9). Order margin versus other orders of similar grade.

problem and found a practical answer to it. One can discover with good reason that the ontology cleared the formulating and modeling of the grade concept.

The study showed that due to the ambiguity of the grade concept it is worth using the order line as the integrator to break down the many to many relations between the grade concept variables. Another significant observation was that the consumption, cost and profitability data is worth saving on the lowest possible level i.e. the customer roll from which they can easily be cumulated to any grade variant and variant combination. The usage of customer roll as the basic data level also absorbs the problems caused by the vagueness of the grade concept. The solution for the calculation of the product profitability described in the article can be broaden up to any paper mill that produces web; in this web means paper, cardboard or plastic web, which is as wide as the machine used and is rolled around the iron or tube as a machine roll.

It will take further studies to create ontological approach for the Meta data in product profitability calculations, which could clarify the numerical figures' history in the reports after each action. This way one would improve the reliability of the reports and traceability of the figures.

\section{REFERENCES}

[1] J. Mattila, "Analysis and utilization of process measurement data with product mix optimization in the paper mill, Licentiate Thesis" (in Finnish), Department of Computer Science, University of Kuopio, Finland, 2006.

[2] V. Sugumaran and V.C. Storey, "Ontologies for conceptual modeling: their creation, use, and management", Data \& Knowledge Engineering, vol. 42, pp. 251-271, 2002.
[3] R. Ungvary and T. Radnai, "Thesaurus and ontology: a formalism of generic inheritance of conceptual characteristics", in Proceedings of Intelligent Engineering Systems, INES '05, IEEE International Conference, 2005. http://ieeexplore.ieee.org/iel5/10418/ 33083/01555149.pdf?tp=\&isnumber $=\&$ arnumber $=1555149$ August 28, 2008.

[4] R. van Rees, "Clarity in the usage of the terms ontology, taxonomy and classification", in Proceedings of the CIB W78's 20th International Conference on Construction IT, Construction IT Bridging the Distance, Amor, R. (Ed.), Waiheke Island, New Zealand, 23-25 April 2003, IB Report 284, pp. 432-440. http://itc.scix.net/data/ works/att/w78-2003-432.content.pdf August 28, 2008.

[5] R. Studer, V.R. Benjamins and D. Fensel, "Knowledge Engineering: Principles and methods", IEEE Transactions on Data and Knowledge Engineering, vol. 25, pp. 161-197, 1998.

[6] B. Chandrasekaran, J.R. Josephson and V.R. Benjamins, "What are ontologies, and why do we need them?", IEEE Intelligent Systems, vol. 14 no. 1 , pp. $20-26,1999$. http://www.cse.ohio-state.edu/ chandra/What-are-ontologies-and-why-we-need-them.pdf August 28, 2008.

[7] S. Suonuuti, Guide to Terminology. NORDTERM 8, 2nd ed, Tekniikan Sanastokeskus ry, Helsinki (in Finnish), 2001.

[8] P. Soffer and I. Hadar, "Applying ontology-based rules to conceptual modeling: a reflection on modeling decision making", European Journal of Information Systems, vol. 16, pp. 599-611, 2007.

[9] D. Fensel, Ontologies: Silver Bullet for Knowledge Management and Electronic Commerce. 2nd ed., Berlin, Germany: SpringerVerlag, 2003.

[10] Semantic Computing Research (SeCo), 2007, http://www.seco.tkk. fi/services/onki//. August 28, 2008.

[11] E.F. Codd, S.B. Codd, and C.T. Salley, "Providing OLAP to User_Analysts: An IT Mandate", Codd E. F. \& Associates, Technical report, 1993. http://dev.hyperion.com/resource_library/white_ papers/providing_olap_to_user_analysts.pdf August 28, 2008.

[12] J. Fogelholm, "Cost Function Modelling in the Paper Industries", Helsinki University of Technology, Otamedia 102, Finland, 2000. 